\section{Female factor infertility}

Abbreviations: PGD, preimplantation genetic diagnosis; $\mathrm{IVF}$, in vitro fertilization; FSH, follicle stimulating hormone; LH, luteotropic hormone; ART, assisted reproductive technology; GIFT, gamete intra fallopian transfer; ZIFT, zygote intra fallopian transfer; ICSI, Intra cytoplasmic sperm injection or microinjection

\section{Short communication}

Infertility is defined as the inability of the couple to conceive or the inability of the mother to lead the fetus to term. The measure of infertility varies greatly according to the criterion used. Some considers that we can need a diagnosis of infertility after a couple tried without success to conceive for only six months, while others argue that the couple must have been unable to conceive for at least two years of frequent unprotected sex. For a woman becomes pregnant, an event chaining is necessary. Her body, and more precisely her ovaries, must first produce a cell, the oocyte, which circulates to the uterus. In the presence of a spermatozoon, fertilization may occur at the beginning of the oviduct (near the ovary). Sperm can survive for 72 hours in the female reproductive tract and the egg must be fertilized within 24 hours after ovulation. ${ }^{1}$ Following the fusion of these two cells, an egg is formed and then implanted in the uterus, where it will be able to develop. Infertility is considered as a big problem for couples who want to become parents but who cannot. This disability can have significant psychological repercussions. ${ }^{2}$ Infertility is very common since it would affect between $10 \%$ to $15 \%$ of couples. For example, the US Centers for Disease Control and Prevention (CDC) confirms that nearly 1 in 10 women will have difficulty getting pregnant. 80 to $90 \%$ of women become pregnant in 1 year and $95 \%$ in 2 years. Even if the data are missing, it seems that more and more women are having difficulties getting pregnant Environmental or infectious factors could be responsible for this evolution. Overweight is also pointed out. It must also be known that fertility decreases with age. ${ }^{3,4}$ However, women are expecting their first child more later, which could also explain why infertility problems are more and more common. In women, a variety of factors can be at the origin of fertility problems. ${ }^{5,6}$ The menstrual cycle is a complex phenomenon, and difficulties can arise in various places. Due to hormonal disorders having normally a key role in the ovulation (see below), it can happen that no ovule reaches maturity and that ovulation, simply, is not done (anovulation, can occur to women with polycystic ovaries), or that the eggs do not come out from the ovary when ripe. In the case of luteal insufficiency, everything goes well until ovulation, but there are complications during the luteal phase, during which the endometrium prepares for implantation of the embryo. An obstruction or dysfunction of the cilia in the trunk, or adhesions in the abdominal cavity (endometriosis, following an operation or infection) can hinder the smooth transfer of the egg (fertilized) to the uterus. Even endometriosis without adhesions can reduce a woman's fertility. Fertility problems can also be the consequence of the fact that the implantation in the uterus has failed (because of a myoma, inflammation of the endometrium ...). Abnormalities in the cervix can also impede the passage of sperm and thus cause a decrease in fertility in women. Finally and recently, Glycosylphosphatidylinositolanchored proteins on the egg surface have been found to play a role in gamete fusion on the basis of in vitro experiments and the loss
Volume 3 Issue I - 2018

\section{Sajida Sboui,' Ahmed Tabbabi \\ 'Faculty of Medicine of Monastir, Monastir University, Tunisia ${ }^{2}$ Department of Hygiene and Environmental Protection, Ministry of Public Health, Tunisia}

Correspondence: Department of Hygiene and Environmental Protection, Ministry of Public Health, Tunisia, Email tabbabiahmed@gmail.com

Received: April 13, 2018| Published: April 23, 2018

of the GPIanchored complement of plasma membrane proteins could prevent fusion. ${ }^{7,8}$ For treating infertility, the proper functioning of the women's reproductive organs is verified. First, the doctor makes sure that the menstrual cycle is normal. Blood tests to check the amount of hormones present make sure that the woman is ovulating well. A hysterosalpingography allows visualizing well the uterine cavity and the fallopian tubes. This examination enables, thanks to the injection of a contrast product, to detect a possible blockage at the level of the tubes. ${ }^{9}$ A laparoscopy, an operation that can visualize the inside of the abdomen and therefore, the ovaries, fallopian tubes and uterus, can be prescribed in case of suspicion of infertility. It can help to detect endometriosis. A pelvic ultrasound can also detect abnormalities of the uterus, tubes or ovaries. Genetic testing may be necessary to detect a genetic origin for infertility.

Many women are unable to conceive and deliver a healthy baby because of genetic factors resulting from hereditary chromosomal abnormalities or defect on a single gene. In fact, infertility can occur if one or both of the future parents have a chromosomal abnormality. Genetic problems can be transmitted to the baby and cause spontaneous abortion because embryos with chromosomal abnormalities have low implantation rates in the mother's uterus. However, if an abnormal embryo becomes implanted, the pregnancy can still result in a miscarriage or the baby can be born with physical, mental or developmental problems. There are several types of chromosomal abnormalities including mainly incomplete chromosomes, inverted chromosome or DNA sequence mutations. A very common genetic cause of infertility is the attachment of a part of a chromosome to another chromosome, i.e. a genetic translocation. Although a parent with translocation is often normal and healthy, his embryo may receive too much or too little genetic material. The most common genetic causes of female infertility are quantitative and structural chromosomal aberrations as well as fragile $\mathrm{X}$ syndrome. Other very common genetic causes of infertility in women often do not show any apparent symptoms. They are diagnosed only by performing genetic tests during blood tests. Although the genetic causes of infertility are untreatable, genetic counseling and diagnosis can determine which type of fertility treatment will provide the best chance of success for example, preimplantation genetic diagnosis (PGD), in vitro fertilization (IVF) or other assisted reproductive technologies. ${ }^{10}$ The decision on the choice of the most appropriate treatment is made during the initial assessment process and may include, depending on the diagnosis, hormone therapy to induce ovulation, surgery 
or medical assistance techniques to procreation, such as in vitro fertilization and similar techniques. The female reproductive cycle is regulated by different hormones released by the endocrine glands. Two of these glands, the hypothalamus and pituitary gland, interact to exert basic control over the release of hormones, including follicle stimulating hormone (FSH) and luteotropic hormone (LH), which play a key role in the process of ovulation. A dysfunction of the hypothalamus or pituitary gland, or problems of interaction between these two glands can induce disorders of ovulation. The induction of ovulation, a treatment based on the administration of fertility factors, aims to correct such hormonal imbalances and thus allows ovulation. Ovulation disorders can also be caused by ovarian failure, when the ovaries do not respond to any stimulation of FSH and LH. There is no treatment for this particular situation, but pregnancy remains possible by donating oocytes or embryos. There are many methods of assisted reproductive technology (ART), including artificial insemination, IVF (In Vitro Fertilization), Gamete Intra-Fallopian Transfer (GIFT), ZIFT (Zygote Intra-Fallopian Transfer or Intratubal Transfer of Zygotes) and ICSI (Intra Cytoplasmic Sperm Injection or Microinjection). These techniques commonly involve the stimulation of multiple follicular developments. Through the use of drugs such as gonadotropins, the ovaries are stimulated to produce more than one mature oocyte at a time. Multiple follicular developments allow physicians to collect multiple oocytes in the patient's ovary. IVF, GIFT, ZIFT and ICSI are distinguished by where fertilization takes place (in vitro or in vivo) and by the stage of cell division at which the fertilized oocytes are implanted in the mother's uterus. ${ }^{11}$

\section{Acknowledgements}

None

\section{Conflict of interest}

The authors declare that they have no conflict of interest.

\section{References}

1. Pei J, Strehler E, Noss U, et al. Quantitative evaluation of spermatozoa ultrastructure after acupuncture treatment for idiopathic male infertility. Fertil Steril. 2005;84(1):141-7

2. Slama R, Ducot B, Keiding N, et al. La fertilité des couples en France. Bulletin Épidémiologique Hebdomadaire. 2012; 87-91.

3. Chavarro JE, Rich Edwards JW. Dietary fatty intakes and the risk of ovulatory infertility. Am J Clin Nutr. 2007;85(1):231-7

4. Löwy I. L'âge limite de la maternité : corps, biomédecine et politique. Mouvements. 2009;59:102-112.

5. Thonneau P, Marchand S, Tallec A, et al. Incidence and main causes of infertility in a resident population $(1,850,000)$ of three French regions (1988-1989). Human Reproduction. 1991;6(6):811-816.

6. Mazuy M. Avoir un enfant: être prêts ensemble. Revue des Sciences Sociales. 2009;41:30-41.

7. Jennifer A, Alfieri, Arlan D, et al. Infertility in female mice with an oocytespecific knockout of GPI-anchored proteins. Journal of Cell Science. 2003;116(11):2149-2155.

8. Klaus Reinhardt, Anne Cecile Ribou. Females become infertile as the stored sperm's oxygen radicals increase. Scientific reports. 2013;3:2888.

9. Régnier-Loilier A. Avoir des enfants en France. Désirs et réalités. Paris, INED, Coll. Les Cahiers de 1. 2007; 279.

10. Bachelot A, De Mouzon J, Adjiman M. La fécondation in vitro : un parcours qui reste difficile, In: de La Rochebrochard E, editor. De la pilule au bébé éprouvette. Choix individuels ou stratégies médicales. 2008;243-261.

11. Thompson C. Making parents. The ontological choreography of reproductive technologies. Cambridge: MIT Press. 2005; p. 360. 